\title{
Crescimento, morfologia radicular e liberação de compostos orgânicos por plântulas de soja em função da atividade de alumínio na solução do solo de campo natural
}

\author{
Growth, root morphology and organic compounds released by soybean seedlings as a function \\ of aluminum activity in a field soil solution
}

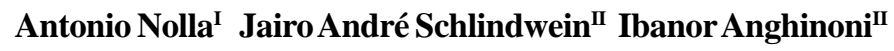

\section{RESUMO}

A toxicidade do alumínio em solos é considerada uma das maiores limitações para a produção das culturas. No entanto, algumas plantas são capazes de tolerar altas concentrações de alumínio por sua complexação com ácidos orgânicos exsudados pelas raizes. No intuito de estudar os efeitos concomitantes de faixas $\mathrm{pH}$ e de aluminio no desenvolvimento radicular de plântulas de soja, desenvolveuse um trabalho na solução de um Argissolo Vermelho distrófico típico de campo natural. Cultivou-se, em câmara de crescimento, plântulas pré-germinadas de soja durante três dias dentro de tubos de ensaio aerados contendo $40 \mathrm{ml}$ da solução do solo $\left(4,2 \mu \mathrm{mol}\right.$ de $\left.\mathrm{Al} \mathrm{L}^{-1}\right)$, submetidos a quatro

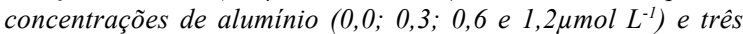
niveis de $\mathrm{pH}(4,0 ; 5,0$ e 6,0). O crescimento radicular e da parte aérea da soja foi menor em condições mais ácidas ( $p H$ 4,0). Em condições de acidez intermediária ( $\mathrm{pH} \mathrm{H}_{2} \mathrm{O} \mathrm{5,0)}$, o crescimento radicular foi menos afetado pelas espécies rizotóxicas de alumínio, mesmo quando submetidas a elevadas concentrações de alumínio na solução do solo.

Palavras-chave: especiação de alumínio, carbono orgânico solúvel, $\mathrm{pH}$ da solução do solo.

\section{ABSTRACT}

Aluminum toxicity in soils is considered one of the major limitations to crop production. However, some plants are able to tolerate high aluminum concentrations due to its complexation with root organic acids exudates. The simultaneous effect of $\mathrm{pH}$ ranges and aluminum concentration in soil solution on soybean seedlings root development was studied in a Rhodic Paleudult soil solution under natural grasses vegetation. Soybean seedlings were grown in growth chamber for three days in aerated test tubes containing $40 \mathrm{ml}$ soil solution

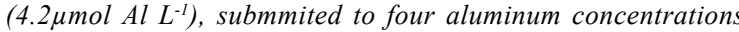

(0.0, 0.3, 0.6 and $\left.1.2 \mathrm{mmol} \mathrm{L}^{-1}\right)$ and three $\mathrm{pH}$ levels (4.0, 5.0 and 6.0). Root and aerial growth decreased under more acidic conditions ( $p H$ 4.0). Root growth was less affected by rhyzotoxic aluminum species at pH 5.0, even when submitted to high concentration of aluminum to the soil solution.

Key words: chemical speciation, soluble organic carbon, soil solution $\mathrm{pH}$.

\section{INTRODUÇÃO}

Os solos brasileiros são, em sua maioria, ácidos, apresentam teores de alumínio em níveis fitotóxicos e baixos teores de cálcio e magnésio trocáveis, características desfavoráveis ao desenvolvimento da maioria das culturas (SOUSA \& LOBATO, 2004), porque causam desordens fisiológicas provocadas pela indisponibilidade ou desbalanço de nutrientes (BARBER, 1995).

A toxicidade do alumínio é uma das maiores limitações para a produção de culturas. À medida que o pH do solo diminui, aumenta a atividade do alumínio na solução do solo e, conseqüentemente, ocorre potencialização dos efeitos nocivos e deletérios desse elemento às culturas (SALET et al., 1999). O sintoma inicial e mais dramático da toxidez do alumínio é a inibição da expansão celular das raízes (TAYLOR, 1988), o que ocasiona redução e engrossamento do sistema radicular, diminuindo a absorção de água e de nutrientes.

'Universidade Federal de Uberlândia (UFU), Instituto de Ciências Agrárias, Av. Amazonas, s/n, bloco 4C, sala 4C127, B, Umuarama, CP 593, 38400-902, Uberlândia, MG, Brasil. E-mail: nolla73@hotmail.com. Autor para correspondência.

"Departamento de Solos, Universidade Federal do Rio Grande do Sul (UFRGS), Av. Bento Gonçalves, 7712, CP 776, 91540-000, Porto Alegre, RS, Brasil. 
Algumas plantas são capazes de tolerar altas concentrações de alumínio $\left(>0,15 \mu \mathrm{mol} \mathrm{L}^{-1}\right)$. O mecanismo de tolerância envolve a detoxificação desse elemento fora da raiz, através de uma reação de complexação com ácidos orgânicos exsudados. Estes são excretados pelo sistema radicular das plantas, devido à exposição ao alumínio (estresse biótico), sendo a quantidade liberada proporcional à concentração externa de alumínio na solução do solo. Essa exsudação radicular é maior no início do crescimento das plantas (STROBEL, 1999). Uma vez liberados, os ácidos orgânicos formam complexos com o alumínio na solução do solo rizosférico, tornando as raízes mais tolerantes ao alumínio (JONES, 1998). Os ácidos orgânicos alteram o pH rizosférico com intensidade variável conforme a espécie vegetal (STROBEL et al., 1999), e a condição de estresse (JONES, 1998), e são estimulados por microrganismos que metabolizam esses compostos (MARSCHNER, 1995; JONES, 1998). Dependendo das condições de solo e da espécie de planta que está sendo utilizada, esses exsudatos promovem alterações diferenciadas no $\mathrm{pH}$ rizosférico, o que promove alteração na espécie iônica de alumínio presente em solução (SALET et al., 1999; FRANCHINI et al., 2001). Em pH baixo, o alumínio livre $\mathrm{Al}(\mathrm{H} 2 \mathrm{O}) 63+$ é a espécie predominante e, segundo PARKER et al. (1988), considerada tóxica para a maioria das plantas. Assim, a tolerância das plântulas ao alumínio e o pH da solução do solo são importantes fatores que devem ser estudados em conjunto.

As leguminosas, principalmente a soja, têm sido utilizadas como plantas indicadoras da toxidez de alumínio (MUZILLI et al., 1976; MARSCHNER, 1995), por serem mais sensíveis à sua presença na solução do solo, com as respostas mais drásticas dessa cultura a essa toxidez se manifestando no estádio plantular (HANSON \& KAMPRATH, 1979). Este trabalho foi desenvolvido com o intuito de verificar os efeitos concomitantes de faixas de alumínio e de $\mathrm{pH}$ na atividade do alumínio na solução do solo e no desenvolvimento de plântulas de soja.

\section{MATERIAL E MÉTODOS}

Coletou-se um volume de um Argissolo Vermelho distrófico típico representativo (10 subamostras) de uma área de campo natural, na profundidade de $0-10 \mathrm{~cm}$, localizado na Estação Experimental da UFRGS (Eldorado do Sul - RS), cuja caracterização química está apresentada na tabela 1 .

Após a coleta, o solo foi umedecido com água destilada até capacidade de campo, esboroado com as mãos, peneirado (peneira de malha de $2 \mathrm{~mm}$ ) e colocado em colunas de PVC de $100 \mathrm{~mm}$ de diâmetro por $200 \mathrm{~mm}$ de comprimento, 24 horas antes da extração da solução do solo. A solução do solo foi extraída pelo método do deslocamento por uma solução de tiocianeto de potássio (KCNS) a 0,5\% (ADAMS, 1974), filtrandoa, posteriormente, com papel Whatmann 42. A solução de solo foi homogeneizada e armazenada sob refrigeração.

Na solução do solo extraída (concentração original de $4,2 \mu \mathrm{mol}$ de $\left.\mathrm{Al} \mathrm{L}^{-1}\right)$, foram aplicados quatro tratamentos de alumínio $\left(0,0,0,30,0,60\right.$ e $1,20 \mathrm{mmol} \mathrm{L}^{-1}$ de $\mathrm{Al})$, em $40 \mathrm{ml}$ da solução dentro de tubos de ensaio (50ml), mantidos sob três níveis de $\mathrm{pH}(4,0,5,0$ e 6,0$)$. A seguir, instalou-se o bioensaio, cultivando-se, em câmara de crescimento, duas plântulas de soja, prégerminadas em caixa de areia, por tubo (fixadas por uma lâmina de isopor), com radículas com $2,5 \mathrm{~cm}$ da cultivar "BR-16", considerada sensível à toxidez de alumínio (MENOSSO et al., 2000). O bioensaio foi conduzido durante três dias, com aeração permanente (bomba de aquário), em um delineamento inteiramente casualisado, com três repetições. $\mathrm{O}$ pH dos tratamentos foi ajustado diariamente, utilizando-se soluções diluídas de $\mathrm{HCl}\left(0,02 \mathrm{mmol} \mathrm{L}^{-1}\right)$ e $\mathrm{NaOH}\left(0,02 \mathrm{mmol} \mathrm{L}^{-1}\right)$.

Avaliou-se a matéria seca e a fresca do sistema radicular e da parte aérea e o comprimento das raízes (medidas com régua) das plântulas de soja. $\mathrm{O}$ raio radicular foi estimado pela fórmula descrita por BARBER (1995): $\mathrm{r} 0=(\mathrm{V} / \mathrm{L} . \pi)^{1 / 2}$; admitindo-se que densidade radicular $=1 \mathrm{~g} \mathrm{~cm}^{-3}$, em que: $\mathrm{r}_{0}=$ raio da raiz (cm) $\mathrm{V}=$ volume da raiz $\left(\mathrm{g} \mathrm{cm}^{-3}\right)$ e $\mathrm{L}=$ comprimento

Tabela 1 - Caracterização química do Argissolo Vermelho distrófico típico sob campo natural utilizado para a extração da solução do solo.

\begin{tabular}{|c|c|c|c|c|c|c|c|c|c|c|c|c|}
\hline \multirow{2}{*}{$\mathrm{pH}\left(\mathrm{H}_{2} \mathrm{O}\right)^{*}$} & \multirow{2}{*}{$\mathrm{pH}(\mathrm{SMP})^{*}$} & $\mathrm{Ca}^{*(1)}$ & $\mathrm{Mg}^{*(1)}$ & $\mathrm{Al}^{*(1)}$ & \multirow{2}{*}{$\begin{array}{c}\mathrm{P}^{*(2)} \\
\mathrm{mg} \mathrm{kg}^{-1}\end{array}$} & \multirow{2}{*}{$\begin{array}{c}\mathrm{K}^{*(2)} \\
\mathrm{mmol}_{\mathrm{c}} \mathrm{kg}^{-1}\end{array}$} & $\mathrm{SB}^{*(3)}$ & $\mathrm{H}+\mathrm{Al}^{*(4)}$ & $\mathrm{T}^{*(5)}$ & $\mathrm{V}^{*(6)}$ & $\mathrm{m}^{*(7)}$ & \multirow{2}{*}{$\begin{array}{c}\operatorname{COS}^{(8)} \\
\mathrm{g} \mathrm{L}^{-1}\end{array}$} \\
\hline & & \multicolumn{3}{|c|}{ 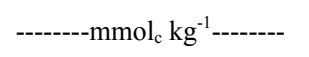 } & & & -------- & $-\mathrm{mmol}_{\mathrm{c}} \mathrm{kg}$ & 1------ & ----------' & --------- & \\
\hline 5,0 & 5,9 & 27 & 14 & 7 & 3,3 & 3,5 & 44,5 & 39 & 83,5 & 53 & 13,59 & 13,9 \\
\hline
\end{tabular}

*Metodologias de análises descritas em Tedesco et al. $(1995){ }^{(1)}=$ Extrator $\mathrm{KCl} 1 \mathrm{~mol} \mathrm{~L}^{-1}$; ${ }^{(2)}=$ Extrator Mehlich I, ${ }^{(3)} \mathrm{SB}$ : soma de bases $(\mathrm{Ca}+$ $\mathrm{Mg}+\mathrm{K}) ;{ }^{(4)} \mathrm{H}+\mathrm{Al}=$ acidez potencial - extrator acetato de cálcio $0,5 \mathrm{~mol} \mathrm{~L}-1$ a pH 7,$0 ;{ }^{(5)} \mathrm{T}=$ capacidade de troca de cátions $(\mathrm{pH} 7,0)=\mathrm{SB}+$ $\mathrm{H}+\mathrm{Al} ;{ }^{(6)} \mathrm{V}=$ saturação por bases $=100 \mathrm{SB} / \mathrm{T} ;{ }^{(7)} \mathrm{m}=$ saturação por alumínio $=100 \mathrm{Al}^{+3} / \mathrm{SB}+\mathrm{Al}^{+3} ;{ }^{(8)} \mathrm{COS}=$ carbono orgânico solúvel (Moore, 1985). 
radicular $(\mathrm{cm})$. O teor de carbono orgânico solúvel da solução do solo foi determinado segundo MOORE (1985). A atividade de alumínio livre na solução do solo foi determinada pelo método do eletrodo de íon seletivo de flúor, descrito em MIYAZAWA et al. (1992). Os resultados foram submetidos à análise de regressão pelo programa SANEST.

\section{RESULTADOS E DISCUSSÃO}

O aumento da concentração de alumínio resultou em aumento, linear em duas condições de acidez ( $\mathrm{pH} 4,0$ e 5,0) e quadrático em outra ( $\mathrm{pH}$ água $6,0)$, do teor de carbono orgânico solúvel, que foi, no entanto, dependente do $\mathrm{pH}$ da solução do solo (Figura 1a), sendo maior no menor $\mathrm{pH}$. Isto porque, segundo JONES (1998), as raízes passam a aumentar a produção de exsudatos (ácidos orgânicos, açúcares, aminoácidos, fenólicos) em quantidade diretamente proporcional à atividade externa de alumínio. Uma vez liberados da raiz, os ácidos orgânicos podem alterar o $\mathrm{pH}$ rizosférico, inativando algumas formas de alumínio, pela formação de complexos na solução do solo. No entanto, é importante observar que o aumento da concentração de alumínio na solução somente aumentou a sua atividade como $\mathrm{Al}^{3+}$ livre $\left(\mathrm{Al}\left(\mathrm{H}_{2} \mathrm{O}\right)_{6}{ }^{3+}\right)$, na condição mais ácida ( $\mathrm{pH} 4,0)$, chegando a se elevar em até 15,8 vezes (Figura 1b). Já nas condições menos ácidas (pH 5,0 e 6,0), a atividade de alumínio, que era relativamente baixa, tornou-se praticamente nula com o aumento de sua concentração inicial, pelo efeito de pH. Com o seu aumento, a atividade de $\mathrm{H}^{+}$diminui e aumenta a atividade de $\mathrm{OH}^{-}$; como resultado, também diminui a atividade de Al livre e aumenta a atividade de
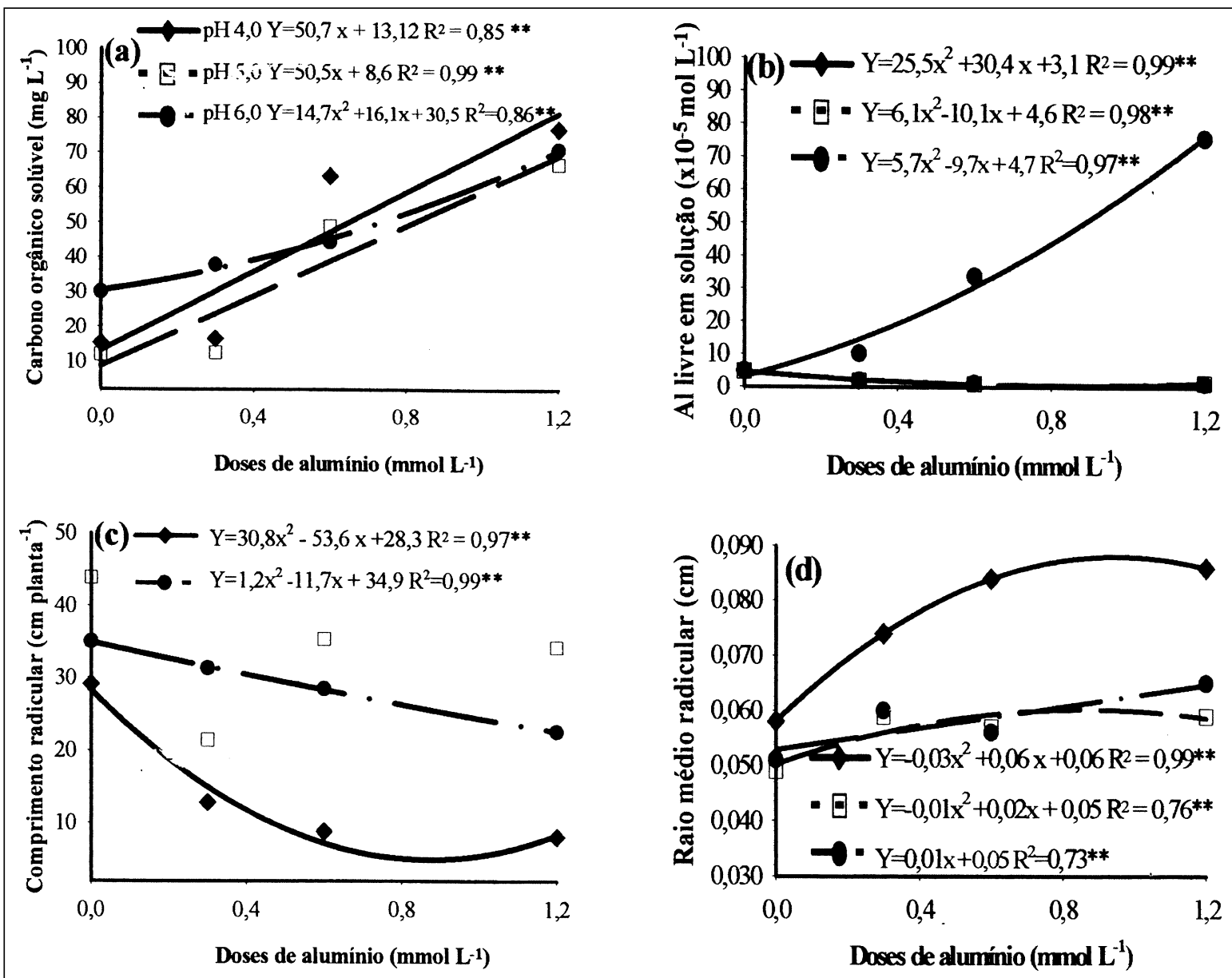

\section{$\neg \mathrm{pH} \mathrm{4,0} \quad-\boxminus-\mathrm{pH} 5,0 \rightarrow \mathrm{pH} \mathrm{6,0}$}

Figura 1 - Carbono orgânico (a), alumínio na solução (b), comprimento (c) e raio médio de raízes (d) de plântulas de soja na solução de um Argissolo Vermelho distrófico típico, sob diferentes níveis de pH e de alumínio.

Ciência Rural, v.37, n.1, jan-fev, 2007. 
$\mathrm{Al}(\mathrm{OH})^{2+} \mathrm{e} \mathrm{Al}(\mathrm{OH})^{2+}$, espécies cada vez menos tóxicas às raízes, respectivamente. Assim, a atividade relativa de $\mathrm{Al}^{3+}$, que é de $100 \%$ em $\mathrm{pH} 4,0$, diminui para somente $50 \%$ em pH 5,0, chegando próxima a $0 \%$, em pH 6,0 (MOORE, 1974).

Como conseqüência disso, o crescimento radicular das plântulas de soja foi menor em pH 4,0 e diminuiu em uma proporção maior com o aumento da concentração inicial de alumínio, quando comparado às demais faixas de $\mathrm{pH}$ (Figura 1c). Isso indica que em $\mathrm{pH} 4,0$, em que a espécie de $\mathrm{Al}\left(\mathrm{H}_{2} \mathrm{O}\right)_{6}{ }^{3+}$ (de maior fitotoxicidade) é predominante em solução (SALET et al., 1999; STROBEL et al., 1999; SCHLINDWEIN et al., 2003), há uma maior redução no crescimento do sistema radicular da soja (RITCHEY et al., 1983).

Além de reduzir o crescimento radicular das plântulas de soja, como esperado no $\mathrm{pH}$ mais baixo, o aumento na concentração de alumínio na solução resultou em uma maior espessura das raízes (Figura 1d). Esses resultados concordam com TAYLOR (1988), ao observar que, em condições de elevada acidez e predominância do $\mathrm{Al}\left(\mathrm{H} \mathrm{O}_{2}\right)_{6}^{+3} \mathrm{em}$ solução, há inibição da expansão das células da raiz e ocorre o seu engrossamento.

Em $\mathrm{pH} 5,0$, o crescimento das raízes foi semelhante ao obtido em pH 6,0 (Figuras 1c,d, 2a,b). Esperava-se, no entanto, que em $\mathrm{pH} 5,0$, a atividade de $\mathrm{Al}^{+3}$ livre fosse maior do que em $\mathrm{pH}$ 6,0 (SALET, 1999), pois, em pH (água) menor do que 5,5, ainda está presente a espécie $\mathrm{Al}\left(\mathrm{H}_{2} \mathrm{O}\right)_{6}^{+3}$ (apesar de apresentar baixa concentração em pH água $=5,0$ ), o que poderia reduzir o desenvolvimento vegetal (SOUSA \& LOBATO, 2004). No entanto, o aumento da concentração de alumínio na solução mantida a pH 5,0 resultou em redução da atividade de alumínio (Figura 1b). É provável que, além do efeito do próprio $\mathrm{pH}$, houve complexação dessa espécie de alumínio pelos exsudatos liberados pelo sistema radicular (Figura 1a), como também observado por KOCHIAN (1995); SALET et al. (1999) e SCHLINDWEIN et al. (2003), reduzindo sua atividade em solução, e, conseqüentemente, seu efeito fitotóxico. É de se esperar que, em condições de campo, a complexação do alumínio com ácidos orgânicos em solução possa proporcionar condições para que a soja

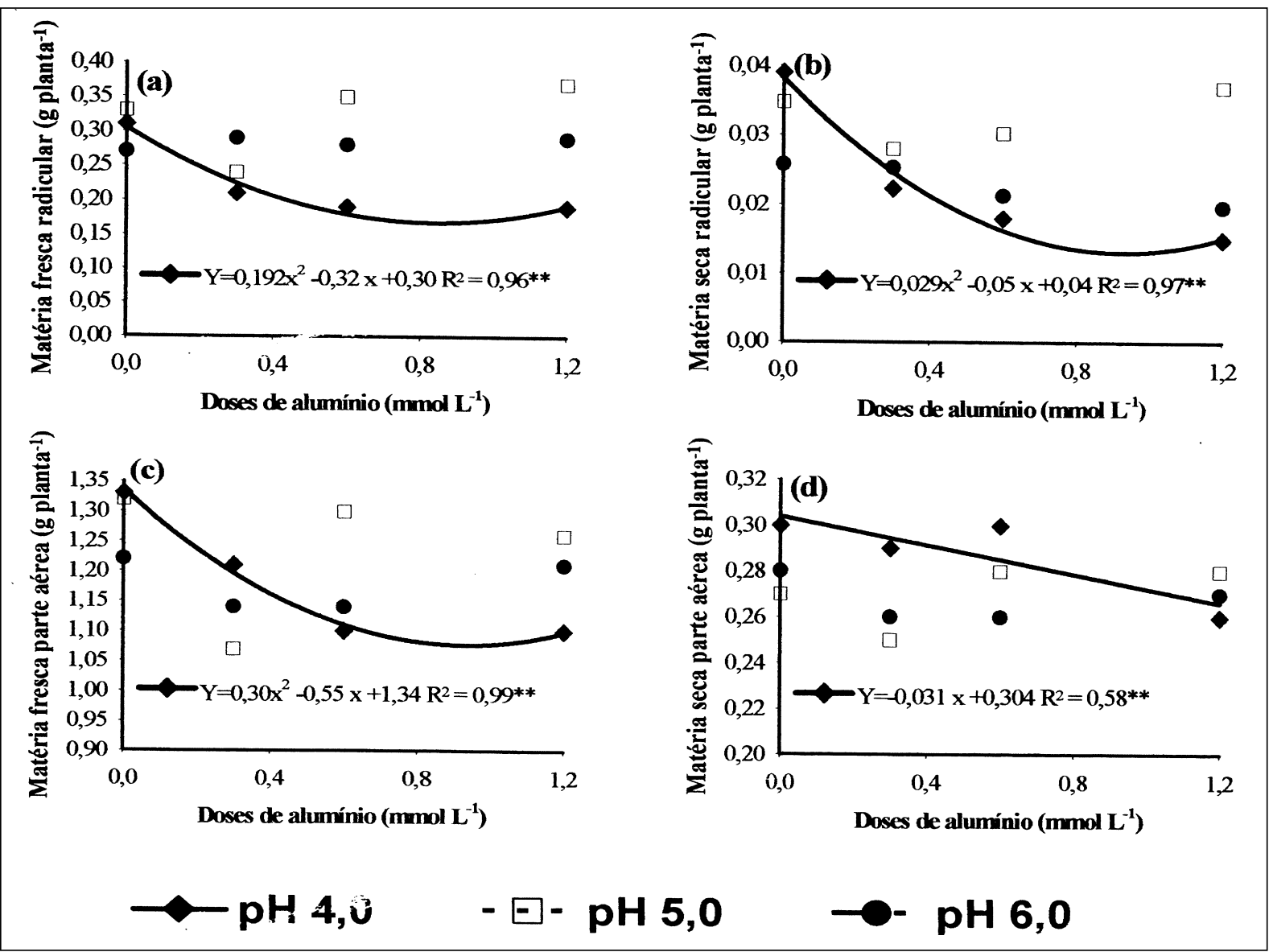

Figura 2 - Matéria fresca (a) e seca (b) do sistema radicular, e matéria fresca (c) e seca (d) da parte aérea de plântulas de soja crescidas na solução de um Argissolo Vermelho distrófico típico, sob diferentes níveis de $\mathrm{pH}$ e de alumínio.

Ciência Rural, v.37, n.1, jan-fev, 2007. 
seja pouco afetada mesmo em pH (água) 5,5 (ANGHINONI \& SALET, 2000). Além disso, é importante observar que, nesse $\mathrm{pH}$, a concentração de espécies de alumínio em solução é baixa no presente trabalho $\left(<1,30 \times 10-5 \mathrm{~mol} \mathrm{~L}^{-1}\right)$, mesmo com elevadas concentrações de alumínio adicionadas à solução $(0,6$ e $\left.1,2 \mathrm{~mol} \mathrm{~L}^{-1}\right)$, o que corresponde ao nível crítico de atividade de alumínio para café (PAVAN \& BINGHAM, 1982) e milho (BRENES \& PEARSON, 1973), justificando a não-redução no desenvolvimento radicular das plântulas de soja.

Em $\mathrm{pH}$ baixo $(\mathrm{pH} 4,0)$, houve redução na matéria seca e fresca das raízes (Figura 2a,b) e da parte aérea (Figura 2c,d), com o aumento da concentração de alumínio na solução. Pode-se inferir que o crescimento da parte aérea da soja possa vir a ser afetado, caso a cultura seja cultivada por um período mais longo, a exemplo do que já ocorrera com o comprimento e a morfologia do sistema radicular (Figura $1 \mathrm{c}, \mathrm{d})$.

\section{CONCLUSÕES}

O crescimento de raízes e da parte aérea é menor na condição mais ácida $(\mathrm{pH} 4,0)$, quando comparado às demais condições (pH 5,0 e 6,0). Em condições de acidez intermediária $(\mathrm{pH} 5,0)$, o crescimento radicular é pouco afetado pelas espécies rizotóxicas de alumínio.

\section{AGRADECIMENTOS}

À Coordenação de Aperfeiçoamento de Pessoal de Nível Superior (CAPES), pela concessão de bolsa ao pesquisador Nolla.

\section{REFERÊNCIAS}

ADAMS, F. Soil solution. In: CARSON, E.W. (Ed.) The plant root and its environment. Charlottesville: University of Virginia, 1974. p.441-485.

ANGHINONI, I.; SALET, R.L. Reaplicação de calcário no sistema plantio direto consolidado. In: KAMINSKI, J. (Coord.) Uso de corretivos da acidez do solo no plantio direto. Pelotas: NRS-SBCS, 2000. p.41-59.

BARBER, S.A. Soil nutrient bioavailability: a mechanistic approach. 2.ed. New York: John Wiley \& Sons, 1995. 414p.

BRENES, E.; PEARSON, R.W. Root responses of three graminae species to soil acidity in Oxisol and Ultisol. Soil Science, Baltimore, v.116, p.295-302, 1973.

FRANCHINI, J.C.L. et al. Rapid transformations of plant water-soluble organic compounds in relation to cation mobilization in an acid Oxisol. Plant and Soil, Dordrecht, v.231, p.55-63, 2001 .

HANSON, W.D.; KAMPRATH, E.J. Selection of aluminum tolerance in soybeans based on seedling root growth. Agronomy Journal, Madison, v.17, p.581-586, 1979.
JONES, D.L. Organic acids in the rhizosphere - a critical review. Plant and Soil, Dordrecht, v.205, p.25-44, 1998.

KOCHIAN, L.V. Cellular mechanisms of aluminum toxicity and resistence in plants. Annual Revision Plant Physiology Mollecular Biology, Bristol, v.46, p.237-260, 1995.

MARSCHNER, H. Mineral nutrition of higher plants. 2.ed. San Diego: Academic, 1995. 889p

MENOSSO, O.G. et al. Tolerância de genótipos de soja ao alumínio em solução nutritiva diluída. Pesquisa Agropecuária Brasileira, Brasília, v.35, p.2157-2166, 2000.

MIYAZAWA, M. et al. Amenização da toxidez de alumínio às raízes do trigo pela complexação com ácidos orgânicos. Revista Brasileira de Ciência do Solo, Campinas, v.16, p.209-215, 1992.

MOORE, D.P. Physiological effects of $\mathrm{pH}$ on roots. In: CARSON, E.W. (Ed). The plant root and its environment. Charlottesville: University of Virginia, 1974. p.135-151.

MOORE, T.R. The spectrophotometric determination of dissolved organic carbon in peat waters. Soil Science Society of American Journal, Madison, v.49, p.1590-1592, 1985.

MUZILLI, O. et al. Tolerância de cultivares de soja e de trigo à acidez do solo. Revista Brasileira de Ciência do Solo, Campinas v.2, p.34-40, 1976.

PARKER, D.R. et al. Aluminum speciation and phytotoxicity in dilute hidroxi-aluminum solutions. Soil Science Society of American Journal, Madison, v.52, p.438-444, 1988.

PAVAN, M.A.; BINGHAM, F.T. Toxicity of aluminum to coffee seedlings grown in nutrient solution. Soil Science Society of American Journal, Madison, v.46, p.993-997, 1982.

RITCHEY, K.D. et al. Relação entre o teor de cálcio no solo e o desenvolvimento de raízes avaliado por um método biológico. Revista Brasileira de Ciência do Solo, Campinas, v.7, p.269-275, 1983.

SALET, R.L. et al. Atividade do alumínio na solução de solo do sistema plantio direto. Revista Científica Unicruz, Cruz Alta, v.1, p.9-13, 1999.

SCHLINDWEIN, J.A. et al. Redução da toxidez de alumínio em raízes de soja por culturas antecessoras no sistema plantio direto. Revista Brasileira de Agrociência, Pelotas, v.9, n. 1, p.85-88, 2003.

SOUSA, D.M.; LOBATO, E. Cerrado: correção do solo e adubação. 2.ed. Brasília: Embrapa Informação Tecnológica, 2004. 416p

STROBEL, B.J. et al. Low molecular weight aliphatic acids in soil solution under different vegetations determined by capillary zone electrophoresis. Plant and Soil, Dordrecht, v.212, p.115121,1999

TAYLOR, G.J. The physiology of aluminum phytotoxicity. In: SIEGAL, H.; SIEGAL, A. (Eds). Metals ions in biological systems. New York: Marcel Dekker, 1988. p.123-163.

TEDESCO, M.J. et al. Análise de solo, plantas e outros materiais. 2.ed. Porto Alegre: Departamento de Solos / UFRGS, 1995. 147p. (Boletim Técnico, 5). 